\title{
How Does Alcohol Consumption Affect People's Income
}

\author{
Zechen $\mathrm{Ye}^{1,{ }^{*}, \mathrm{a}, \dagger}$, Yantong $\mathrm{Zhu}^{2, \mathrm{~b}, \dagger}$, Hang $\mathrm{Liu}^{3, \mathrm{c}, \dagger}$, and Yukun $\mathrm{Bai}^{4, \mathrm{~d}, \dagger}$ \\ ${ }^{1}$ The Chinese University of Hong Kong Shenzhen, School of Management and Economics, 5181722001 Longxiang \\ Boulevard, Shenzhen, China \\ ${ }^{2}$ Jilin University, Business School, 130012 No.2699Qianjin Street, Changchun, China \\ ${ }^{3}$ The University of Manchester, School of Social Science, m139pl Oxford Rd, Manchester, UK \\ ${ }^{4}$ Shanghai Lixin University of Accounting and Finance, School of International Economics and Trade, 200120 \\ No.995Shangchuan Road, Shanghai, China \\ * Corresponding author: 118020497@link.cuhk.edu.cn \\ These authors contributed equally.
}

\begin{abstract}
With the development of society and technology, it has seen a dramatic rise in people's living standards. Wine as a high level of consumer goods in people's daily lives accounts for an increasing proportion. Therefore, the relationship between people's per capita income needs to be studied further. The research using the National Longitudinal Survey of Youth (NLSY) data mainly utilized a multiple linear regression model to create a linear relationship between alcohol behaviors and wages. It introduced a method of the instrumental variable to eliminate the endogenous problems in the model. The drinking behaviors were divided into two groups based on the number of drinks consumed per day, and to make our conclusion more reasonable, samples that have more than 4 drinks per day were dropped in the research. After fixing the model using an instrumental variable, the coefficients of the independent variables are positive and tested to be valid, which means moderate alcohol use positively impacts people's wages. Although we get the results not consistent with other related researches, our conclusion still has some practical implications. Moderate alcohol use is beneficial to the heart and cerebrovascular health, especially to the long-term overtime workers. Still, it alleviates the brain's pressure, which improves cognitive performance and increases the work's productivity. Therefore, drinking alcohol properly is a wise choice, which can keep us healthy and increase our income compared to drinking excessively.
\end{abstract}

Keywords: alcohol consumption, people's income.

\section{INTRODUCTION}

The per capita alcohol consumption in some fastdeveloped country including Europe and the United States, is ahead of other regions, which indicates that with the development of the economy and the improvement of people's income, people will pay more and more attention to quality consumption [1]. The more people earn, the more alcohol they consumed. However, most studies show that illicit and excessive drinking can lead to a series of social problems, such as increased unemployment [2] and high traffic accident rates [3] and a certain impact on people's physical and mental health [4]. To reduce such problems, the United States enacted the Minimum Drinking Age Act in 1984, which raised the minimum drinking age to 21 years old. In the $1990 \mathrm{~s}$, the United States economy showed a good trend of stable development after the reform of the social system. The alcohol market in the United States also shows a stable level of improvement. What does the worker market income change response to moderate alcohol consumption? Some research has shown that moderate drinking is good for health and has a positive effect on personal income in the labor market [5]. Moderate drinking can relax the body and mind and relieve the pressure, becoming a pastime for the workers. Therefore, drinking may indeed have a positive effect on income [6]. Alcohol consumption and income may have a mutually reinforcing effect.

Michael T. French and Gary A. Zarkin have researched the relationship between alcohol use and labor market outcome [7]. In their study, they analyzed the database of more than 1,000 employees at 4 worksites 
to test whether alcohol use affects wages and test for a nonlinear relationship between alcohol consumption and people's income. The test results showed a reverse Ushaped relationship between these two variables, with a peak of about 1.5 to 2.5 drinks per day. It suggests that people, approximately two drinks per day on average, earn more than non-drinkers and alcoholics. Bray and Jeremy $\mathrm{W}$ established a model that eliminated the mediated effect of alcohol use, which actually affects human capital accumulated, on wages [8]. The data is collected from the 1979 cohort of the National Longitudinal Survey of Youth (NLSY), originally designed to follow young adults through their first years in the labor market. This analysis showed that moderate alcohol use has a positive impact on obtaining education and experience, so it positively affects human capital accumulated, which will affect wages. They found that although heavier drinking slightly reduces the wage returns to education and experience, the overall effect of alcohol use on the returns to education and experience was positive but largely insignificant. And the consumption of alcohol has no negative impact on human capital accumulation. Jenny N. LYE and Joe H Irschberg researched the effects of drinking and smoking on wages and test the endogeneity arising from a potential causal relationship between earnings and alcohol consumption [9]. They used The Australian National Household survey in 1995, which aims to collect information about Australians' health status and health-related aspects of their lifestyle. They found that drinking is more beneficial for low/moderate drinkers in terms of wages than for either abstainers or heavy drinkers. Still, there is no significant relationship between alcohol consumption and wages on the heaviest consumers of alcohol.

Similar research has done by Mullahy and Sindelar. They obtained the data of Alcohol Supplement of the National Health Interview Survey in 1988 to analyze how alcohol consumption affects employment and unemployment [10]. They have established a dummy for diagnosed alcohol abuse and two dummies for heavy drinking patterns to measure alcohol consumption and applied the Ordinary Least Squares (OLS) and instrument variables to regress the variables. The main findings showed that problem drinking results in reduced employment and increased unemployment, but the impacts were statistically insignificant. Lastly, Francesco Renna focused on the effects of alcohol abuse and alcoholism on wage rates and work hours [11]. His research is conducted using data for a sample of employed men from the National Longitudinal Survey of Youth-1979(NLSY79). After applying the method of Ordinary Least Squares (OLS), he found that both alcohol abuse and alcoholism have a negative effect on the hours of work. The negative coefficient of OLS indicates that alcoholism reduced the annual work time by 69 hours.

\section{METHOD}

\subsection{Data description}

The analysis presented in this essay is conducted using data from the National Longitudinal Survey of Youth (NLSY). It includes information on labor market outcomes, alcohol consumption, and assorted demographics for individuals in each of 2 years - 1989 and 1994 . We further restricted the data to young adults between the ages of 24 and 32 in 1989 (and hence 29-37 in 1994). Each individual has a unique identifier (variable named id), and the year is indicated by the variable named year. Since our purpose is to analysis how alcohol consumption affects the outcome of the labor market, variables like total wage and salary income in the past calendar year and the total number of hours worked in the past calendar year should be included in the data for analysis. For alcohol measuring, a categorical variable was used to indicate the number of times in the past month the individual has had 6 or more drinks(drnk6m) in one sitting. Another categorical variable that we have generated is the drink days, and it indicates the number of days in the last month the individual has had at least 1 drink (drinkday). If an individual has a drink less than 3 days, we assign the variable DRINKDAY equals 1 to 5 by dividing 5 equal standards, and each standard includes 3 days. Lastly, for those who have to drink more than half a month, we set the DRINKDAY equals 6. Making the conclusion persuasive, samples were selected if its year equals 1989 and dropped if its wgsal (total wage and salary income in the past calendar year) is 0. Lastly, we generated a categorical variable extenddrink which number is 0 to 5 , and its numbers are the reverse of variable gtint (whether the individual has ever drunk more than intended). If gtint is 5 and extenddink is 0 , gtint equals 4 , and extenddrink equals 1 , and so forth.

\subsection{OLS and Instrument variable}

The multiple linear regression model and instrumental variable (IV) were mainly used to study in our research. We established the multiple linear regression model to describe the relationship between the dependent variables lgwgsal (log of total income in the past calendar year) and wgperh (income per hour in the past calendar year) and the independent variables drink6m, drinkday, and extenddrink.

$$
Y=\beta_{0}+\beta_{1} \operatorname{drink} 6 m+\alpha \mathbf{X}+\mathrm{u}
$$

Drink6m can be replaced as drinkday and extenddrink. $\mathrm{X}$ is the control variable.

Multiple linear regression model is to measure the relationship between the independent and dependent variables under the condition of controlling other factor variables unchanged. 
To eliminate the endogenous problem in the model, we introduced an exogenous variable, an exogenous variable, $\mathrm{z}$.

First stage:

$$
\text { drink6m vhat }=\gamma_{0}+\gamma_{1} Z+\alpha \mathbf{X}+\mathrm{v}
$$

Second stage:

$$
Y=\beta_{0}+\beta_{1} \text { drink6m vhat }+\alpha \mathbf{X}+\mathrm{u}
$$

Drink6m can be replaced as drinkday and extenddrink. $\mathrm{X}$ is the control variable.

And the $\mathrm{z}$ satisfies two conditions that it should be strongly correlated with the independent variable but should not be correlated with the error term. Therefore, we chose famsize (a categorical variable of famsz which represents the number of people in the individual's family) as instrumental variable. And here, the two-stage least square method was utilized to address the endogenous problem.

\section{RESULT}

We first established six basic multiple linear regression models to analyze the relationship between alcohol consumption and people's income. We got OLS estimate coefficients of them, which can be noticed in Table1. These models were dividing into two groups, one takes lgwagsal as a dependent variable, and the other y is wgperh. We used 15 control variables, including age, sex, race, health, education, unemployment rate, family information like siblings, net income of the family, and if parents worked when children at 14 and so forth. To make our conclusion more reasonable, the samples whose value of the variable perday (the average number of drinks per day on a day when individual drinks) is larger than 4 should be dropped. Because we agreed that drinking excessively definitely affects individual's working condition. Therefore, considering that most human beings drink while having meals. With higher salaries, people are likely to work late, which means they may have an extra meal or just drink at night, the number 4 was chosen as a proper maximum of perday. The results shown in Table1 indicate that these models are not reasonable as most of the p-values here are too high. To improve our models to be more valid, we thought our models might have an endogenous problem, so famsize was introduced as an instrument variable. After testing the models have endogenous problems and the instrument is not weak, the two-stage least square method was used to estimate coefficients again and obtain results in Table2. From the results, it could be clearly seen that all the coefficients are positive, and the p-values are conformed to a $95 \%$ confident interval, so we concluded that moderate alcohol use has a positive impact on people's wages.
Table1. Result of multiple linear regression

\begin{tabular}{l|llll}
\hline & lgwgsal & \multicolumn{3}{l}{ wgperh } \\
\cline { 2 - 5 } & $\begin{array}{l}\text { Coef. } \\
\text { (Std.Err.) }\end{array}$ & $\begin{array}{l}\text { P- } \\
\text { value }\end{array}$ & $\begin{array}{l}\text { Coef. } \\
\text { (Std.Err.) }\end{array}$ & $\begin{array}{l}\text { P- } \\
\text { value }\end{array}$ \\
\hline drnk6m & -0.004 & 0.702 & -0.041 & 0.766 \\
& $(0.011)$ & & $(0.139)$ & \\
drinkday & 0.035 & 0.000 & 0.132 & 0.152 \\
& $(0.007)$ & & $(0.092)$ & \\
extenddrink & -0.001 & 0.955 & 0.248 & 0.067 \\
& $(0.010)$ & & $(0.136)$ & \\
\hline
\end{tabular}

Table2. Result of two stages least square

\begin{tabular}{l|llll}
\hline & lgwgsal & \multicolumn{3}{l}{ wgperh } \\
\cline { 2 - 5 } & $\begin{array}{l}\text { Coef. } \\
\text { (Std.Err.) }\end{array}$ & $\begin{array}{l}\text { P- } \\
\text { value }\end{array}$ & $\begin{array}{l}\text { Coef. } \\
\text { (Std.Err.) }\end{array}$ & $\begin{array}{l}\text { P- } \\
\text { value }\end{array}$ \\
\hline drnk6m & 0.574 & 0.000 & 4.681 & 0.002 \\
& $(0.135)$ & & $(1.532)$ & \\
drinkday & 0.275 & 0.000 & 2.268 & 0.001 \\
& $(0.057)$ & & $(0.709)$ & \\
extenddrink & 0.435 & 0.000 & 3.868 & 0.003 \\
& $(0.118)$ & & $(1.349)$ & \\
\hline
\end{tabular}

\section{DISCUSSION}

In this essay, we used the National Longitudinal Survey of Youth (NLSY) data to derive the long-term relationship between alcohol consumption and the income of labor. Our result shows that alcohol consumption has a positive effect on labor income. This may not be consistent with the conclusions from similar research. Our purpose is to analyze the correlation between alcohol consumption and labor income but, more importantly, by analyzing the data, we try to find out whether moderate and excessive drinking have different effects on income. Using the data for those who drink less than 4 times per day, the regression result indicates a positive effect on earnings. This indicates a pivotal point in the relationship between alcohol consumption and labor income. Therefore, we use 4 times per day as a marker to distinguish between moderate drinking and heavy drinking. However, this distinction may not apply to the real world because it determines heavy drinking or moderate drinking, and it is the health conditions of the drinkers that matter and make the distinction. For our study's purpose, to analyze the effect of alcohol consumption on income, we set any alcohol consumption greater than 4 times per day as heavy drinking and less than 4 times as moderate drinking.

The result suggested that moderate drinking can improve labor income. Since the labor income is determined by labor productivity, it can conclude that 
moderate drinking has a positive effect on productivity. So, what is the rationale behind it? Although excessive alcohol consumption has severe negative health consequences, moderate consumption will increase blood flow to the brain, helping prevent cognitive decline and improve brain health. Especially in the case of longterm overtime workers, appropriate drinking can eliminate fatigue to improve work efficiency. Research done by $\mathrm{C}$ Baum-Baicker suggested that low alcohol doses improve certain types of cognitive performance included problem-solving and short-term memory [12]. Therefore, those benefits associated with moderate drinking may increase labor productivity and thus their income. Our result has further validated this view. Therefore, this result can be recommended as a reference for enterprises to establish a policy on alcohol consumption. By understanding that appropriate alcohol consumption can improve work efficiency, firms can allow employees to drink appropriately at work. Especially for some employees who work overtime, taking a small amount of alcohol can help eliminate fatigue and improve labor productivity.

However, our result is not without some qualification. First, we are using the incidents of drinking per day for our alcohol measurements, but this may not reflect the actual alcohol intake per day. Although we have measured the number of drinks per day, the alcohol content may be different for different alcohol types. For example, the alcohol content for a standard beer in the United States is about $5^{\circ} \mathrm{ABV}$ (Alcohol by volume), while for wine, it is $12^{\circ}$. The highest is the distilled spirit which can reach $50^{\circ} \mathrm{ABV}$. Therefore, the alcohol content of people who drink beer per day may be quite different from those who drink distilled spirits. This may distort the distinction between moderate drinking and binge drinking. Hence, people who drink 4 times per day may not all have the same alcohol content. It is estimated that beer is the most common type of alcohol consumed in the United States, contributing about $45 \%$ by volume consumed from the year 1985 to 1990 , followed by spirit, at just less than $40 \%$ [1]. Since the ratio of these two alcoholic drinks consumption is not that different, our result may further be distorted by the type of alcohol consumption. Besides, the data did not consider the amount of alcohol consumed per drinking, which can also affect our judgments of moderate and excessive drinking. For example, the number of glasses per drink and the size of glasses used by people can significantly affect the amount of alcohol consumed, which can also affect our distinction of moderate and excessive drinking.

\section{CONCLUSION}

This paper used the data from the National Longitudinal Survey of Youth (NLSY), filtered and processed it. It dropped the value of drinking more than
4 drinks per day, which we considered excessive drinking, leaving the values that less than 4 drinks, that is, moderate drinking. Also, multiple regression models and control variables were adopted to investigate the relationship between alcohol consumption and workforce income. In addition, to eliminate the endogenous problem, an instrumental variable was introduced into the model.

The result is similar to those of related studies. It found that moderate drinking (less than 4 drinks per day) has a positive effect on income, though the results have some limitations, such as different alcohol content and different type of wine. Drinking too much not only takes a toll on the body but also affects work efficiency. Moderate drinking, by contrast, is healthier, not only improving cognition but also reducing stress and increasing productivity, which in turn leads to higher earnings. Therefore, it's important to know that moderate alcohol consumption is a good alternative to teetotal and excessive drinking.

\section{REFERENCES}

[1] World Health Organization. (2019). Global status report on alcohol and health 2018. World Health Organization.

[2] Ruhm, C. J. (1995). Economic conditions and alcohol problems. Journal of health economics, 14(5), 583-603.

[3] Skog, O. J. (2001). Alcohol consumption and mortality rates from traffic accidents, accidental falls, and other accidents in 14 European countries. Addiction, 96(1s1), 49-58.

[4] Adrian, M., \& Barry, S. J. (2003). Physical and mental health problems associated with the use of alcohol and drugs. Substance use \& misuse, 38(1113), 1575-1614.

[5] Lee, J. H. (2013). The effect of moderate drinking in income and health. Journal of Industrial Economics and Business, 26(4), 1857-1887.

[6] Auld, M. C. (2005). Smoking, drinking, and income. Journal of Human Resources, 40(2), 505518.

[7] French, M. T., \& Zarkin, G. A. (1995). Is moderate alcohol use related to wages? Evidence from four worksites. Journal of health economics, 14(3), 319344.

[8] Bray, J. W. (2005). Alcohol use, human capital, and wages. Journal of Labor Economics, 23(2), 279. 312 . 
[9] Lye*, J. N., \& Hirschberg, J. (2004). Alcohol consumption, smoking and wages. Applied Economics, 36(16), 1807-1817.

[10] Mullahy, J., \& Sindelar, J. (1996). Employment, unemployment, and problem drinking. Journal of health economics, 15(4), 409-434.

[11] Renna, F. (2008). Alcohol abuse, alcoholism, and labor market outcomes: looking for the missing link. ILR Review, 62(1), 92-103.

[12] Baum-Baicker, C. (1985). The health benefits of moderate alcohol consumption: a review of the literature. Drug and alcohol dependence, 15(3), 207-227. 